\title{
Histone acetyltransferase and Polo- like kinase 3 inhibitors prevent rat galactose-induced cataract
}

\author{
Fumito Kanada ${ }^{1}$, Yoshihiro Takamura ${ }^{2}$, Seiji Miyake ${ }^{2}$, Kazuma Kamata ${ }^{1}$, Mayumi Inami ${ }^{1}$, \\ Masaru Inatani ${ }^{2} \&$ Masaya $\mathrm{Oki}^{1,3^{*}}$
}

Diabetic cataracts can occur at an early age, causing visual impairment or blindness. The detailed molecular mechanisms of diabetic cataract formation remain incompletely understood, and there is no well-documented prophylactic agent. Galactose-fed rats and ex vivo treatment of lenses with galactose are used as models of diabetic cataract. To assess the role of histone acetyltransferases, we conducted cataract prevention screening with known histone acetyltransferase (HAT) inhibitors. Ex vivo treatment with a HAT inhibitor strongly inhibited the formation of lens turbidity in high-galactose conditions, while addition of a histone deacetylase (HDAC) inhibitor aggravated turbidity. We conducted a microarray to identify genes differentially regulated by HATs and HDACs, leading to discovery of a novel cataract causative factor, Plk3. Plk3 mRNA levels correlated with the degree of turbidity, and Plk3 inhibition alleviated galactose-induced cataract formation. These findings indicate that epigenetically controlled Plk3 influences cataract formation. Our results demonstrate a novel approach for prevention of diabetic cataract using HAT and Plk3 inhibitors.

Most cataracts are age-related, and nearly $100 \%$ of people develop cataracts by 80 years of age ${ }^{1}$. Diabetes is also a major cause of cataracts, and patients with diabetes develop cataracts earlier and approximately five times more frequently ${ }^{2}$. As the global incidence of diabetes continues to increase, the incidence of diabetic cataracts will increase and become a major cause of early-onset visual impairment. The only available cataract treatment is surgical removal of the crystalline lens and replacement with a intraocular lens, but this approach is associated with significant adverse effects, and is not available in remote locations. Therefore, extending the time to required lens replacement is clinically desirable, but there are currently no known pharmacological interventions for diabetic cataract $^{3}$.

Increased intracellular osmotic pressure due to accumulation of polyol and lens epithelial cell (LEC) apoptosis are major contributing pathologies of diabetic cataract. High intracellular osmotic pressure promotes lens turbidity by increasing cellular swelling and subsequent lens cortex degradation ${ }^{4,5}$, which can be prevented by inhibiting polyol accumulation using aldose reductase inhibitors ${ }^{6}$. On the other hand, addition of hydrogen peroxide to induce LEC apoptosis also causes cortical cataracts and ultimate collapse of the lens cortex ${ }^{7}$. Prior studies have also demonstrated that polyol accumulation increases LEC apoptosis, suggesting that these factors are interrelated ${ }^{8}$. Although the cellular events contributing to diabetic cataract formation have been reported ${ }^{4-6,8}$, the detailed molecular mechanisms for these events remain incompletely understood. Elucidating the molecular events of cataract formation is important to identify therapeutic interventions to prevent or delay diabetic cataract formation.

DNA sequence-independent gene expression regulation mechanisms, collectively known as epigenetics, regulate diverse physiological and pathological phenomena, and epigenetic regulators may therefore be viable therapeutic targets for many disease processes ${ }^{9}$. Epigenetic modifications are regulated in part by DNA methylation and posttranslational histone modification, both of which can either repress or promote gene transcrip$\operatorname{tion}^{10}$. In age-related cataract, hypermethylation-mediated transcriptional repression of the antioxidant enzyme Gstm3 promoter region has been confirmed and may contribute to cataract formation ${ }^{11}$. In diabetic cataract, the antioxidant inhibitor Keap1 is transcriptionally activated by hypomethylation of the promoter region ${ }^{12}$. Further,

${ }^{1}$ Department of Applied Chemistry and Biotechnology, Graduate School of Engineering, University of Fukui, Fukui, 910-8507, Japan. '2Department of Ophthalmology, Faculty of Medical Sciences, University of Fukui, Fukui, 910-1193, Japan. ${ }^{3}$ Life Science Innovation Center, University of Fukui, Fukui, 910-8507, Japan. *email: ma4sa6ya@u-fukui. ac.jp 
(a)

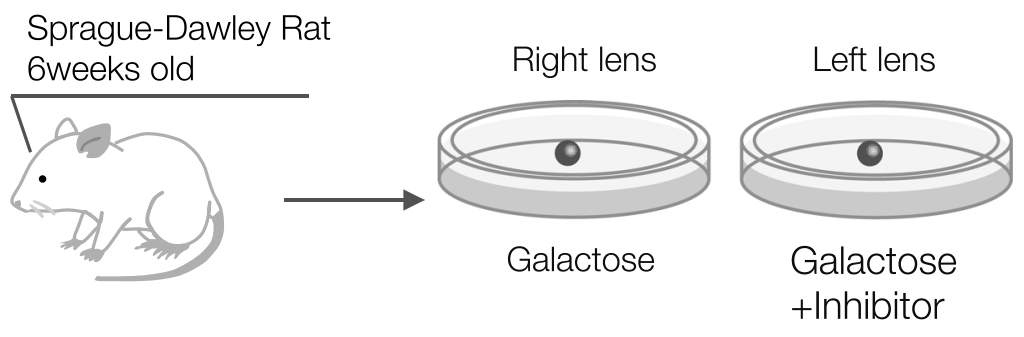

(b)

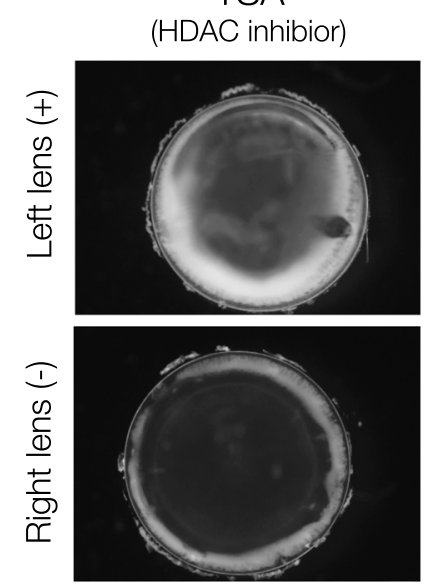

(c)
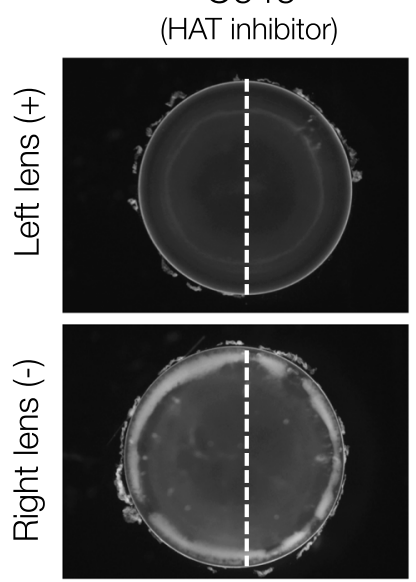
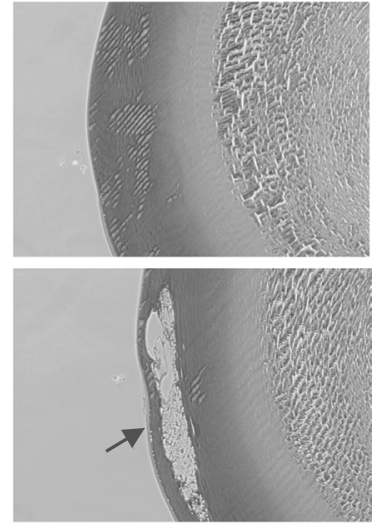

Figure 1. HAT inhibition alleviates galactose-induced cataract formation. (a) Diagram of ex vivo experiment. The inhibitor was added to the left lens (with the right lens serving as a Ctrl) and incubated for 4 days in medium containing $30 \mathrm{mM}$ galactose. (b) Representative photomicrograph of lenses on Day 4 after addition of $0.8 \mu \mathrm{M}$ TSA or vehicle. (c) Representative histological photographs of lenses at $40 \mu \mathrm{M}$ C646 (Day 4). Lenses tissue sections cut in the direction of the broken line were stained with hematoxylin-eosin. Only the right lens (Ctrl group) shows large vacuoles, indicated by arrows.

methylation rates of Cryaa, Ercc6, and Ogg1 are also changed in cataracts ${ }^{13-15}$. Additionally, posttranslational histone modification, especially by acetylation, may also be associated with cataract formation. Generally, histone acetyltransferase relaxes the chromatin structure through histone acetylation, increasing expression of neighboring genes. HATs are classified according to functional roles and structural features, including Pcaf and Gcn5 in the GNAT family, Tip60 and Moz in the MIST family, and p300 and Cbp in the p300/Cbp family ${ }^{16}$. Several prior studies investigated the relationship between histone acetylation and other types of cataract formation. UV exposure is a known risk for cataracts, and UV-B irradiation of LECs induces histone deacetylation ${ }^{14}$. Rong et al. reported that when the rabbit lens was treated with the HAT inhibitor anacardic acid, white turbidity formed on the lens surface ${ }^{17}$. Moreover, this turbidity did not form when anacardic acid and the histone deacetylase (HDAC) inhibitor Trichostatin A (TSA) were applied in co-treatment. Tgf- $\beta$ induced cataracts in rats also causes lens surface turbidity, which is similarly inhibited by TSA ${ }^{18,19}$. These reports suggest that epigenetic modifications are involved in cataract formation, and that preventing these modifications suppresses cataract formation. However, the roles of epigenetic modifications in diabetic cataract are unknown.

In the present study, to evaluate the preventive effects of HAT and HDAC inhibitors in rat diabetic-like cataracts, ex vivo lens turbidity was induced by incubation in galactose-containing media. We then evaluated whether HDAC/HAT inhibitors affected lens turbidity in this model. Subsequently, we used microarray analysis of rat lenses \pm HAT/HDAC inhibitors to identify the target gene Plk3, which contributes to galactose-induced cataract formation. These results identified a novel mechanism of HAT regulation of glycated cataract through Plk3.

\section{Results}

HAT inhibitors prevent diabetic-like cataracts. We initially investigated the effect of HDAC inhibitors on lens turbidity to assess the role of histone acetylation in diabetic-like cataract formation. The rat crystalline lens was cultured in galactose-containing medium as a diabetic cataract model (Fig. 1a). The galactose cataract model is suitable for inhibitor screening because cataract formation occurs rapidly and reliably. When lenses were cultured in medium containing $30 \mathrm{mM}$ galactose for 4 days, cortical cataract formation occurred, and was further increased by addition of the HDAC inhibitor TSA (Fig. 1b). This suggested a relationship between histone acetylation and lens turbidity, prompting us to next determine the effect of HAT inhibitors on cataract formation. We tested 26 different inhibitors targeting various HATs (Table 1$)^{20-31}$, and found that 16 of the 26 tested inhibitors decreased cataract formation (Supplementary Fig. S1). The HAT inhibitors embelin and ECGC are slightly cloudy, but we determined that these inhibitors prevented cataract formation because lens turbidity was 


\begin{tabular}{|c|c|c|c|c|c|}
\hline Name & Target & Place of purchase & Concentraion & $\begin{array}{l}\text { Prevent } \\
\text { effect }\end{array}$ & Source \\
\hline CTK7A & p300/PCAF & EMD Millipore (USA) & $100 \mu \mathrm{M}$ & 0 & $(20)$ \\
\hline Garcinol & p300/PCAF & Abcam (UK) & $2 \mu \mathrm{M}$ & 0 & $(20,21)$ \\
\hline Anacardic Acid & p300/PCAF & Abcam (UK) & $20 \mu \mathrm{M}$ & O & $(20,21)$ \\
\hline MG149 & TIP60/MOZ & Selleck Chemicals (USA) & $50 \mu \mathrm{M}$ & O & $(22)$ \\
\hline C646 & $\mathrm{p} 300$ & Sigma Aldrich (USA) & $40 \mu \mathrm{M}$ & 0 & $(20,21)$ \\
\hline СРTH2 & GCN5 & Cayman Chemical (USA) & $80 \mu \mathrm{M}$ & O & $(23)$ \\
\hline gallic acid & HAT & Sigma Aldrich (USA) & $100 \mu \mathrm{M}$ & 0 & $(24)$ \\
\hline $\begin{array}{l}\text { (-)-Epigallocatechin gallate } \\
\text { (ECGC) }\end{array}$ & HAT & Wako (Japan) & $50 \mu \mathrm{M}$ & 0 & $(20,21)$ \\
\hline EML425 & $\mathrm{p} 300 / \mathrm{CBP}$ & Tocris (UK) & $200 \mu \mathrm{M}$ & O & $(20)$ \\
\hline ISOX DUAL & CBP/BRD4 & Sigma Aldrich (USA) & $20 \mu \mathrm{M}$ & O & $(25)$ \\
\hline Plumbagin & p300 & Sigma Aldrich (USA) & $2 \mu \mathrm{M}$ & 0 & $(20,21)$ \\
\hline TH1834 & TIP60 & Axon Medchem (Netherlands) & $50 \mu \mathrm{M}$ & O & $(20)$ \\
\hline windorphen & HAT & Sigma Aldrich (USA) & $40 \mu \mathrm{M}$ & O & $(26)$ \\
\hline Remodelin & NAT10 & Cayman Chemical (USA) & $40 \mu \mathrm{M}$ & 0 & $(27)$ \\
\hline Embelin & PCAF & Abcam (UK) & $40 \mu \mathrm{M}$ & O & $(20)$ \\
\hline СВР30 & p300 & Cayman Chemical (USA) & $5 \mu \mathrm{M}$ & O & (20) \\
\hline Curcumin & $\mathrm{p} 300 / \mathrm{CBP}$ & Abcam (UK) & $40 \mu \mathrm{M}$ & $x$ & $(20,21)$ \\
\hline $\begin{array}{l}\text { 2,6-bis((e)-3-bromo-4- } \\
\text { hydroxybenzylidene) cyclohexan } \\
\text { (HAT inhibitor) }\end{array}$ & p300 & Abcam (UK) & $40 \mu \mathrm{M}$ & $x$ & $(28)$ \\
\hline Epigenetic Multiple Ligand & $\mathrm{p} 300 / \mathrm{CBP}$ & Santa Cruz (USA) & $50 \mu \mathrm{M}$ & $x$ & $(29)$ \\
\hline NU9056 & TIP60 & Santa Cruz (USA) & $100 \mu \mathrm{M}$ & $x$ & $(30)$ \\
\hline Butyrolactone 3 (MB-3) & GCN5 & Abcam (UK) & $200 \mu \mathrm{M}$ & $x$ & $(20,21)$ \\
\hline L002 & $\mathrm{p} 300 / \mathrm{CBP}$ & Cayman Chemical (USA) & $10 \mu \mathrm{M}$ & $x$ & $(20)$ \\
\hline SPV106 & $\mathrm{p} 300 / \mathrm{CBP}$ & Sigma Aldrich (USA) & $50 \mu \mathrm{M}$ & $x$ & $(31)$ \\
\hline Chetomin & HIF1a/p300 complex & Cayman Chemical(USA) & $50 \mathrm{nM}$ & $x$ & $(21)$ \\
\hline Ischemin & p300/CBP & Tocris (UK) & $100 \mu \mathrm{M}$ & $x$ & $(20,21)$ \\
\hline $\begin{array}{l}\text { KG501(Naphthol AS-E } \\
\text { phosphate) }\end{array}$ & $\mathrm{p} 300 / \mathrm{CBP}$ & Sigma-Aldrich (USA) & $50 \mu \mathrm{M}$ & $x$ & $(21)$ \\
\hline
\end{tabular}

Table 1. HAT inhibitor list. The list summarizes the prophylactic effect of 26 HAT inhibitors. Concentration indicates the concentration of the drug used.

significantly reduced compared with the vehicle control. Interestingly, specific inhibitors such as TH1834 (Tip60), Embelin (Pcaf), CPTH2 (Gcn5), and C646 (p300) had similar efficacies. However, some HAT inhibitors were ineffective (Supplementary Fig. S2). To observe the inside of the lens, a section was prepared and stained with hematoxylin and eosin. In the vehicle control (Ctrl) lens exposed to galactose, numerous vacuoles were found in the equator, and the cortex was largely collapsed (Fig. 1c). On the other hand, the p300 inhibitor C646 almost completely prevented tissue collapse and maintained normal lens tissue (Fig. 1c).

To quantitatively evaluate lens opacity, the average value of the brightness of the entire lens was calculated from the micrograph. We used C646 as a representative HAT inhibitor and TSA as an HDAC inhibitor, and measured turbidity daily on Days 1-4 of incubation. The Ctrl group, incubated in normal medium, did not change from Day 1 to Day 4, but turbidity increased in the galactose group in a time-dependent manner (Fig. 2a). There was no significant difference between the galactose, C646, and TSA groups on Day 1 (Fig. 2a). However, from Days 2-4, opacity increased in the galactose and TSA groups in a time-dependent manner, whereas in C646, opacity was similar to that of Day 1 (Fig. 2a). Next, to observe changes in lens luminance distribution, the lens histogram was calculated on Day 4 (Fig. 2b). This is strongly dependent on the strong turbidity of the cortex, and the brightness of the entire lens is illustrated in a single peak. This analysis also suggested that galactose and TSA increased lens opacity, while C646 decreased lens opacity (Fig. 2b). To investigate concentration-dependent effects of C646, we evaluated its effects at varying concentrations, identifying that turbidity gradually decreased from $10 \mu \mathrm{M}$ to $40 \mu \mathrm{M}$ (Fig. 2c). These results indicate that diabetic-like cataract formation is affected by histone acetylation, suggesting that recovery of histone acetylation levels attenuates cataract formation.

Downstream factors controlled by HAT. Our findings suggested that HAT and HDAC might affect cataract formation, so we next sought to determine which downstream mediator(s) may be responsible for the observed effects. To comprehensively assess cataract-mediating factors oppositely regulated by HAT and HDAC, we performed a microarray. First, we created a Venn diagram with a group of genes significantly reduced in the Ctrl and HAT inhibitor $(\mathrm{C} 64640 \mu \mathrm{M})$ groups, and a group of genes significantly increased in the HDAC inhibitor group (TSA $0.8 \mu \mathrm{M}$ ) compared with galactose. There were 72 differentially regulated genes common between C646 and Ctrl, but there were five genes common between TSA and Ctrl, and three between TSA and C646. Plk3 and Loc100362769 were the only genes that were significantly decreased in C646 and Ctrl, and significantly 
(a)

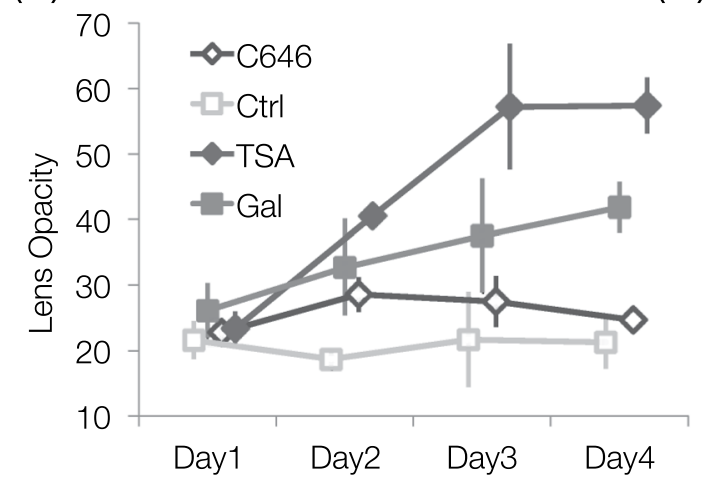

(C)

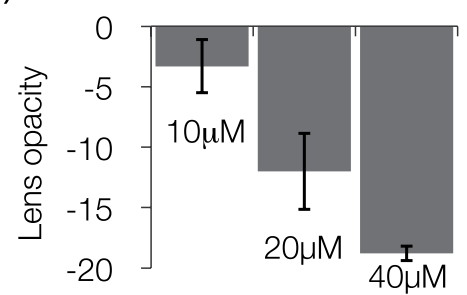

(b)

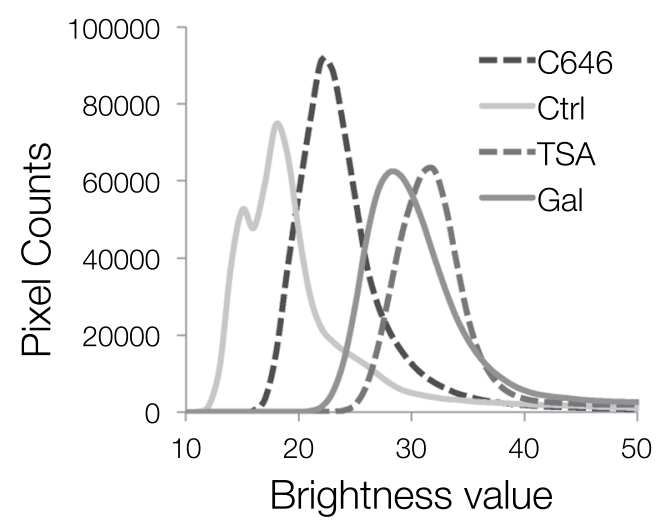

Figure 2. Effects of HAT/HDAC inhibition on lens opacity. (a) Changes in lens opacity over time. Opacity, which is a weighted average of lens brightness values, is sensitive to the effects of strong white turbidity in the cortex. C646 and TSA were added to galactose medium. All lenses on each date were removed from different animals $(n=3$, only galactose is $n=9)$. Galactose-containing medium was used as the Ctrl (right lens). (b) A histogram of luminance values is shown to visualize the opacity of the entire lens. The same lens as Day 4 of Fig. 2a was used. (c) Concentration-dependent decreases in opacity with C646 treatment. The weighted average values of the left lens (inhibitor condition) to the right lens (galactose-only condition) of the same rat were subtracted. The right picture shows the effect of the C646 HAT inhibitor by concentration. The inset is the Ctrl (right) lens. Data are expressed as mean \pm SD.

increased in TSA (Fig. 3). Next, we performed annotation analysis using DAVID (https://david.ncifcrf.gov) to investigate whether the genes related to these two genes were affected by cataract formation. We uploaded the gene group (210) increased in the galactose group relative to the Ctrl group, and extracted the top three clusters with high enrichment scores (Table 2). Apoptosis and FoxO terms in the top 1 and 3 clusters of this list included Plk3.

To confirm the microarray results, $P l k 3$ expression was quantitatively measured by real-time qRT-PCR (Fig. 4a). We measured Plk3 expression in all four groups from Days 1-4, identifying that Plk3 expression increased in the galactose group relative to the Ctrl in a time-dependent manner (Fig. 4a). In the C646 group, Plk3 expression was similar to that of the galactose group on Day 1, but gradually decreased after Day 2 in a time-dependent manner (Fig. 4a). Plk3 expression was higher in the TSA group than in the galactose group at all time points, but this difference was not statistically significant (Fig. 4a). Furthermore, we compared the turbidity of Ctrl and galactose samples with $P l k 3$ expression, revealing a monotonically increasing relationship, suggesting that lens turbidity and Plk3 expression were positively correlated (Fig. $4 \mathrm{~b}$ ). To demonstrate that these phenomena are not ex vivo specific, we performed in vivo experiments (Fig. 4c). As a result, cataracts induced by galactose-containing diets closely resembled those ex vivo and had increased Plk3 expression (Fig. $4 \mathrm{~d}, \mathrm{e}$ ). Taken together, these findings demonstrate that Plk3 is regulated by HAT and HDAC, and is associated with lens turbidity. To determine the causative role of Plk3 in cataract formation, we assessed the effect of Plk3 inhibitor in galactose-induced cataract formation. Cataract formation was decreased by treatment with the Plk3 inhibitor GW843682X (Fig. 5a, left). Plk3 is activated by the DNA damage response factor Atm ${ }^{32}$. Addition of KU55933, an Atm inhibitor, also decreased cataract formation (Fig. 5a, right). Both inhibitors had a similar efficacy to that of C646 (Fig. 5b).

Downstream factors controlled by Plk3 and Atm inhibitors. To investigate the relationships between Hat, Plk3, and Atm inhibitors, we performed microarray analysis. A total of 1201 genes with two-fold or more changes in expression compared to the control (galactose) following treatment with each inhibitor were extracted. The increasing and decreasing patterns of expression in many genes were similar between the three inhibitor treatments (Fig. 6a). Cluster and PCA analyses identified high similarity between the genes inhibited by C646 and GW843682X, but the Ctrl was located in the furthest cluster (Fig. 6a,b). Because there was no significant trend in the overall expression pattern, we sought to determine whether the three inhibitors commonly affected the 


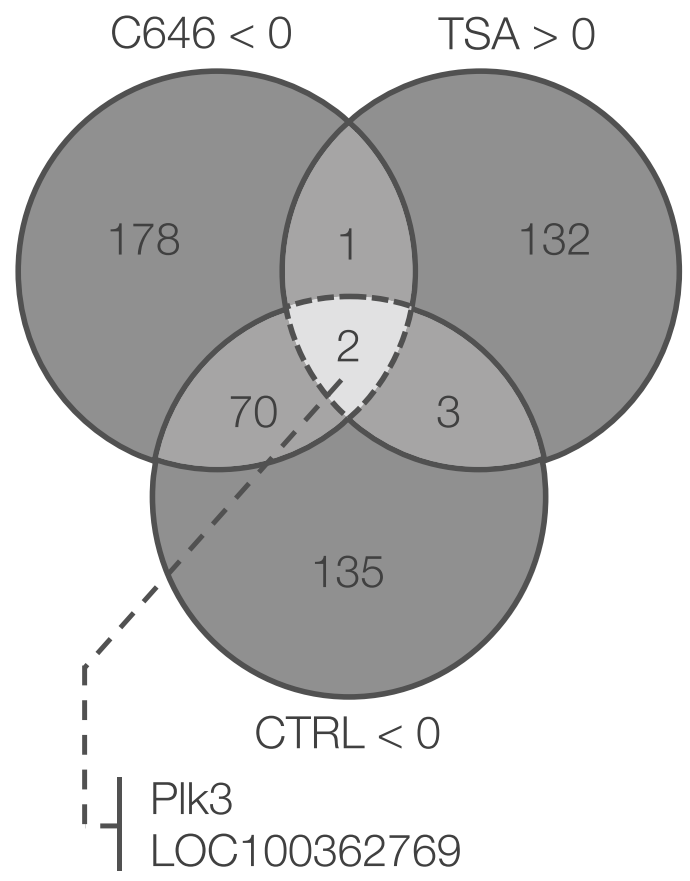

Figure 3. Identification of epigenetically regulated cataract-causing factors by microarray. Venn diagram of fluctuated genes in microarray analysis. C646 and Ctrl show $* P<0.05$ and reduced gene clusters compared with galactose. TSA shows $* P<0.05$ and an increased gene cluster compared with galactose.

\begin{tabular}{|l|l|l|l|l|}
\hline Category & Term & Count & PValue & Fold Enrichment \\
\hline Annotation Cluster 1 & Enrichment Score: 2.28 & & & \\
\hline KEGG_PATHWAY & rno04068:FoxO signaling pathway & 5 & $1.15 \mathrm{E}-03$ & 10.22 \\
\hline KEGG_PATHWAY & rno04115:p53 signaling pathway & 4 & $1.75 \mathrm{E}-03$ & 15.88 \\
\hline KEGG_PATHWAY & rno04110:Cell cycle & 3 & $7.12 \mathrm{E}-02$ & 6.56 \\
\hline Annotation Cluster 2 & Enrichment Score: 2.09 & & & \\
\hline GOTERM_BP_DIRECT & $\begin{array}{l}\text { GO:0010628 positive regulation of } \\
\text { gene expression }\end{array}$ & 7 & $1.65 \mathrm{E}-03$ & 5.42 \\
\hline GOTERM_BP_DIRECT & GO:0042493 response to drug & 7 & $9.17 \mathrm{E}-03$ & 3.81 \\
\hline GOTERM_BP_DIRECT & $\begin{array}{l}\text { GO:0010629 negative regulation of } \\
\text { gene expression }\end{array}$ & 4 & $3.60 \mathrm{E}-02$ & 5.42 \\
\hline Annotation Cluster 3 & Enrichment Score: 1.83 & & & \\
\hline GOTERM_BP_DIRECT & $\begin{array}{l}\text { GO:0043065 positive regulation of } \\
\text { apoptotic process }\end{array}$ & 6 & $5.93 \mathrm{E}-03$ & 5.10 \\
\hline UP_KEYWORDS & Apoptosis & 5 & $1.51 \mathrm{E}-02$ & 5.20 \\
\hline GOTERM_BP_DIRECT & GO:0006915 apoptotic process & 5 & $3.65 \mathrm{E}-02$ & 3.93 \\
\hline
\end{tabular}

Table 2. Annotation Cluster list. Gene ontology analysis of 210 genes whose expression was significantly increased compared with the control. The top three enrichment scores are shown.

expression of a subset of genes. When the heat map in Fig. 6a was replaced with a Venn diagram, 12 genes showed variations in expression between the Ctrl group and each of the three inhibitor groups (Fig. 6c). The results of gene cluster analysis were confirmed by real-time qRT-PCR, and six genes with significant differences in expression irrespective of the inhibitor used were extracted (Fig. 6d). Arid5b, Lif, and Rsad2 had significant differences in expression, suggesting that these genes are common downstream factors of HAT, Atm, and Plk3.

\section{Discussion}

In diabetic cataracts, accumulation of sorbitol increases osmotic stress and apoptosis, disrupting lens homeostasis and ultimately leading to cataract formation ${ }^{4,5}$. However, the detailed molecular mechanisms of diabetic cataract remain incompletely understood. In the present study, we report that HAT inhibition alleviated galactose-induced diabetic-like cataract formation, and demonstrated that histone acetylation correlates with cataract formation. Furthermore, microarray analysis identified that $P l k 3$ was controlled by HAT, and subsequent functional analyses revealed that Plk3 contributed to galactose-induced cataract formation. These findings represent a novel mechanism of epigenetically regulated diabetic cataract formation. 

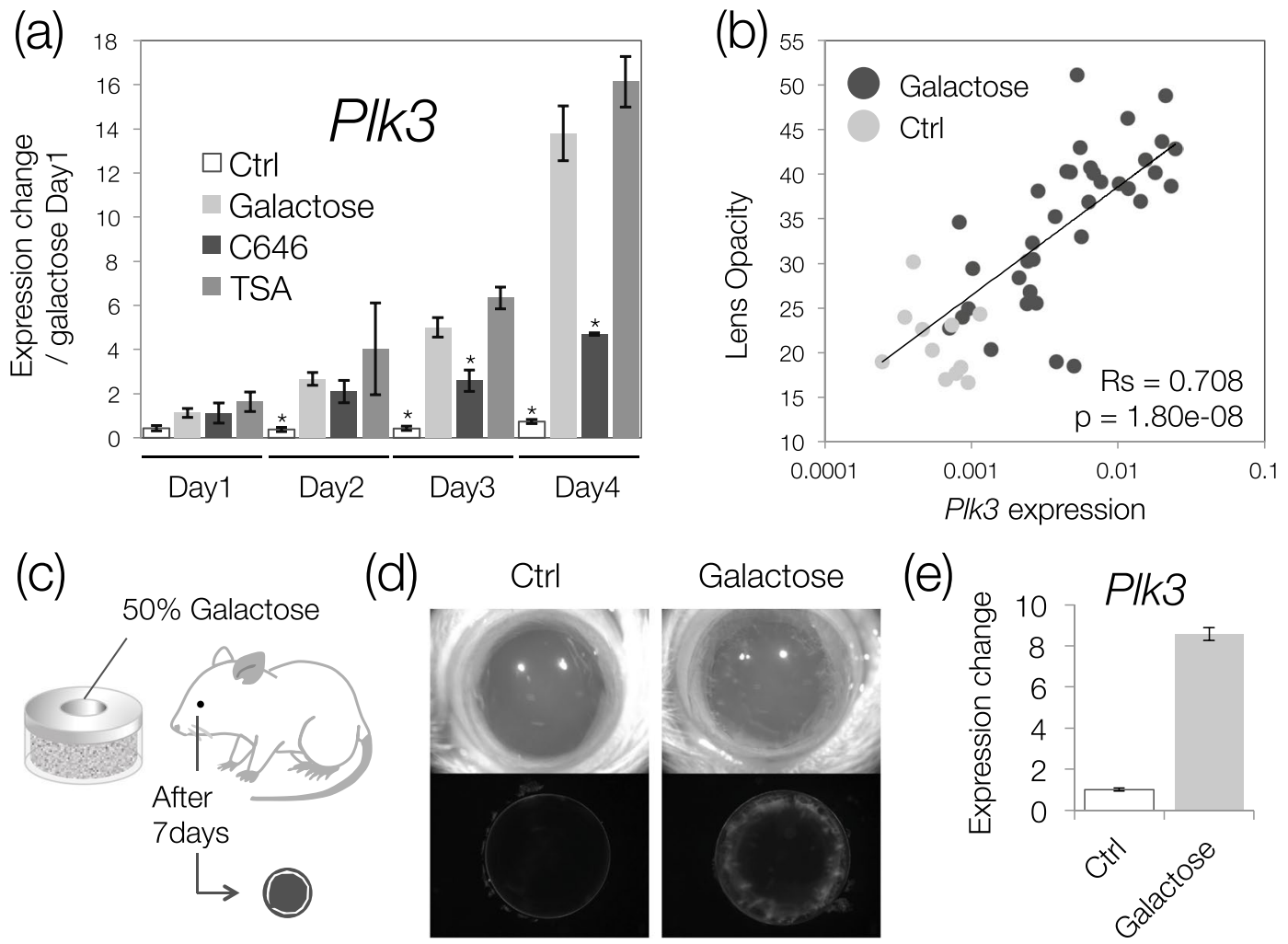

Figure 4. $P l k 3$ expression correlates with cataract severity. (a) $P l k 3$ mRNA expression over time was measured using real-time qRT-PCR $(n=3$ /group, galactose only $n=9)$. C646 and TSA were added to galactose medium. Data are expressed as mean \pm SEM. $* P<0.05$ versus galactose only. (b) The horizontal axis plots the logarithmic value of Plk3 expression level, and the vertical axis plots the degree of lens opacity. Each point used galactose and control from Days 1-4. Rs and $P$ value is cultured Spearman's rank correlation. mRNA levels were normalized to Gapdh expression. (c) Diagram of the in vivo experiment. (d) Photomicrograph of the eye above, and of the lens removed below. (e) mRNA expression in vivo was measured using real-time qRT-PCR $(\mathrm{n}=4)$.

(a)

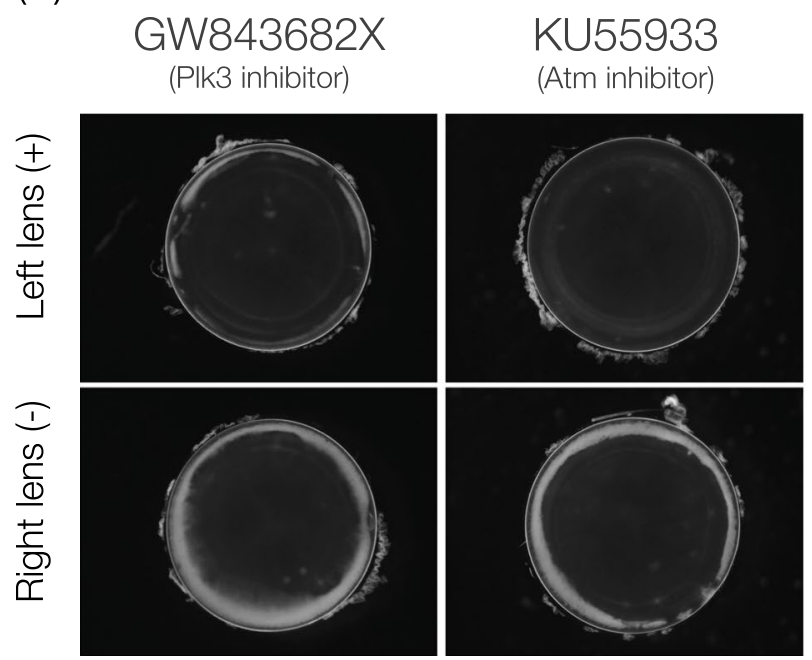

(b)

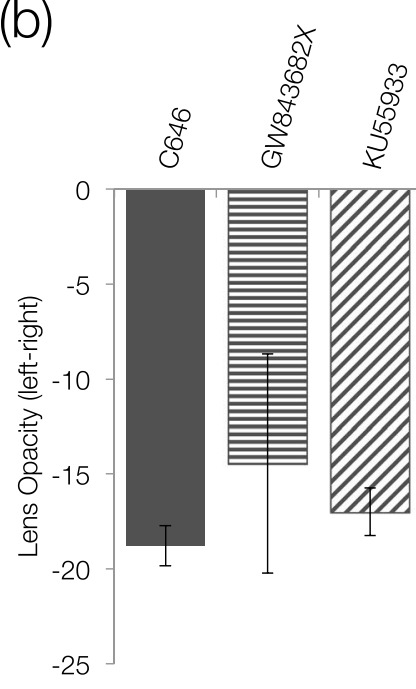

Figure 5. Plk3 and Atm inhibition alleviate galactose-induced cataract formation. (a) Representative photomicrographs of Day 4 lenses after inhibitor addition (Plk3 inhibitor GW843682X $1 \mu \mathrm{M}$, Atm inhibitor KU55933 10 $\mu$ M). GW843682X or KU55933 was added to galactose medium. (b) Comparison of lens turbidity between inhibitors. The values of the left lens (inhibitor condition) to the right lens (galactose-only condition) from the same rat were subtracted. Data are expressed as mean $\pm \mathrm{SD}$. 
(a)

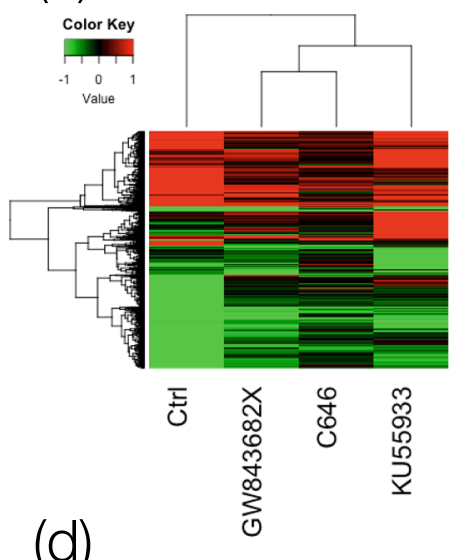

(b)

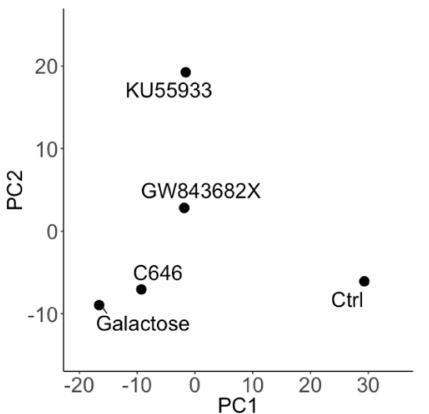

(c)

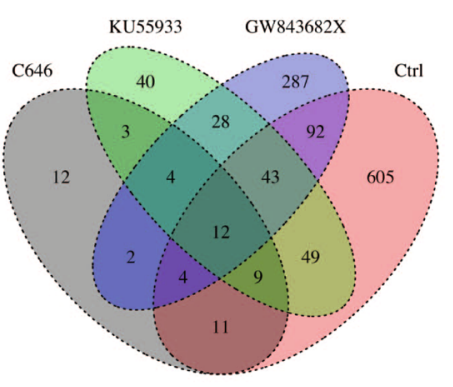

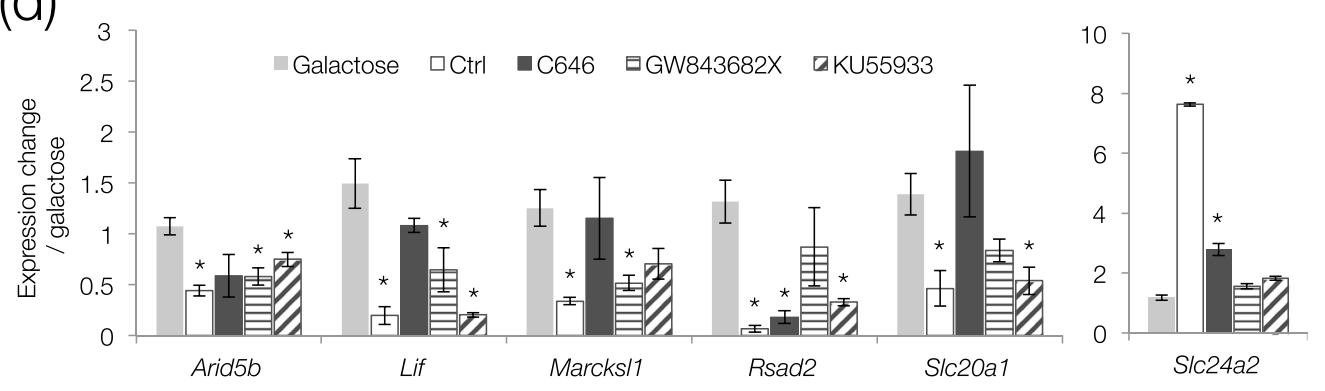

Figure 6. Correlation of HAT, Plk3, and Atm inhibitors. C646, GW843682X, or KU55933 was added to galactose medium. (a) Heat map of genes (1201) with more than a 2-fold variation in any one or more treatment group. (b) Principal component analysis map of 1201 genes. Proximal points in the plot have high expression profile homology. (c) Venn diagram of 1201 genes. In 12 genes that fluctuated more than 2-fold under all conditions, the increasing and decreasing tendencies agreed. (d) Real-time qRT-PCR of six genes (five reduced genes and one increased gene) that showed significant expression changes in any sample compared with galactose. mRNA levels measured were normalized to Gapdh expression levels. Data are expressed as mean \pm SE. $* P<0.05$ versus galactose.

$\mathrm{Plk} 3$ is a serine/threonine kinase, and plays a role in cell cycle progression and tumorigenesis ${ }^{33}$. Plk3 is activated by Atm and causes apoptosis via p53 activation ${ }^{32,33}$. Although no prior studies have reported a direct relationship between Plk3 and cataract, exhaustive gene expression analyses of the murine LEGSKO and Mip mutant cataract models revealed that Plk3 expression was increased in both models compared with $\mathrm{WT}^{34,35}$. Also, some reports indirectly support our hypothesis that Plk3 regulated cataract formation. For example, the cataract-inducing factors $\mathrm{H}_{2} \mathrm{O}_{2}$ and $\mathrm{UV}$ both activate $\mathrm{Plk} 3^{36,37}$. UV-induced cataract is attenuated by caffeine, which also inhibits Plk3 via Atm ${ }^{32,38,39}$. In addition, osmotic stress is also a risk factor for cataract formation, and activates Plk3. Further, Wang et al. revealed that in human corneal epithelial cells osmotically stressed by excess sorbitol, DNA damage occurs and Plk 3 is activated ${ }^{40}$. In diabetic cataracts, osmotic pressure is increased by polyol produced from sugar, and our results demonstrate that Plk3 is up-regulated in the context of galactose-induced diabetic cataract ${ }^{41}$ (Fig. $\left.4 \mathrm{a}\right)$.

Several reports have previously identified that HDAC is activated in age-related and Tgf- $\beta$ induced cataracts, and that cataract formation is alleviated by HDAC inhibition, unlike in the present study ${ }^{14,18,19}$. However, there are no prior reports of HDAC activity is involved in diabetic or galactose-induced cataract formation. In particular, Tgf- $\beta$-induced cataract is caused by multilayered LECs, and the mechanism of cataract formation is fundamentally different ${ }^{42}$. Also, in diabetic retinopathy, histone acetylation is increased, and HDAC activation alleviates the pathology of retinopathy ${ }^{43}$. Therefore, it is possible that ocular histone acetylation increases in diabetes and in ocular diabetic complications. Plk3 may also be controlled by epigenetics in this context.

The tumor suppressor p53 is a transcription factor that binds p300, inducing apoptosis. p53 overexpression not only up-regulates Plk3, but also activates histone acetylation of the Plk3 promoter ${ }^{4}$. However, these findings were obtained in cancer cells, so they may not be relevant to the crystalline lens. Therefore, it will be necessary to confirm this effect of p53 in crystalline lens, and determine whether p53 inhibition has a similar functional effect to that of Plk3 inhibition.

We also sought to determine which factors operate downstream of Plk3, and identified several factors including Arid5b, Lif, and Rsad2 (Fig. 6d). Interestingly, these factors are involved in lipid metabolism, which is surprising because there has been no report so far about a link between lipid metabolism and cataract. Arid5b is a regulator of vascular smooth muscle cell differentiation and proliferation ${ }^{45}$. It complexes with the demethylase $\mathrm{Phf} 2$ to function as an epigenetic determinant of signal sensing by removing repressive histone methylation marks from to its target promoters ${ }^{46}$. Phf2-overexpressing mice develop steatosis, and Arid5b knockout mice 
are resistant to high fat diet-induced weight gain and obesity ${ }^{47,48}$. Lif induces terminal differentiation of myeloid leukemia cells, and inhibits their growth ${ }^{49}$. It also reduces the activity of lipoprotein lipase, an enzyme responsible for triglyceride degradation, through transcriptional regulation ${ }^{50}$. Administration of Lif dose-dependently increases rat serum triglyceride level ${ }^{51}$. Rsad2 is found in a wide range of organisms, and inhibits viral replica$\operatorname{tion}^{52}$. Genotype-phenotype correlations in obese mice using ATR-FTIR spectroscopy has shown that Rsad2 is associated with obesity-related diseases ${ }^{53}$. Additionally, Rsad 2 knockdown mice show increased inflammation of adipose tissue caused by decreased antiviral activity, but are immune to the deleterious effects of high fat diet feeding ${ }^{54}$. Thus, inhibition of Arid5b, Lif, and Rsad 2 may be effective in reducing diabetes-related disorders, such as the impaired homeostasis of lens epithelial cells. Determining the roles of these three factors in lens opacity will require further investigation.

The present study demonstrated for the first time that Plk3 upregulation through HAT may be involved in the onset of diabetic cataract. This suggests that inhibitors of HAT, Plk3, and Atm are potential therapeutic modalities for diabetic cataract. The future work, we will need to find for regulator of HAT and direct regulator of Plk3.

\section{Materials and Methods}

In vivo and Ex vivo assays. Six-week-old male Sprague Dawley rats were purchased from Sankyo Laboratory Service. Rats were euthanized with $\mathrm{CO}_{2}$ asphyxiation, and eyeballs were removed. For Ex vivo experiments, whole lenses were maintained in $2 \mathrm{ml}$ serum-free M199 medium containing $0.1 \% \mathrm{BSA}^{55}$. C646 (Wako), TSA (Wako), GW843682X (AdooQ Bioscience), or KU55933 (Chemscene) were added to the culture medium at the final concentrations of $40,0.8,1$, and $10 \mathrm{mM}$, respectively. All lenses were maintained at $37^{\circ} \mathrm{C}$ in a humidified incubator with $95 \%$ room air and $5 \% \mathrm{CO}_{2}$. Culture medium (Sigma) was renewed every second day throughout the culture period. Lenses were cultured for up to 4 days and photographed using a microscope. For in vivo experiments, rats were fed the following diets for 1 week: normal MF powder (Ctrl), and MF powder plus $50 \%$ galactose (Galactose). The rats were then anesthetized the rats and their eyes were photographed via a microscope. The experiments were approved by Animal Research Committee, University of Fukui (Application number: 28091), and all experiments were performed in accordance with relevant regulations for Animal Research at University of Fukui.

Microscopic observation. Images were captured in the dark using a SZX12 stereomicroscope combined with a DP58 camera (Olympus). At this time, the lens was placed in a $35 \mathrm{~mm}$ dish containing $7 \mathrm{~mL}$ PBS. The degree of opacity was measured as brightness ( 0-255) using ImageJ, and a weighted average was calculated.

RNA extraction, cDNA preparation, and real-time qRT-PCR. After culture, the entire lens was homogenized in TRIzol reagent (Thermo Fisher Scientific) and RNA was extracted according to the manufacturer's instructions. cDNA was synthesized with a reverse transcription kit (Applied Biosystems). For quantitative analysis of mRNA expression, SYBR Green master mix (Applied Biosystems) was used to amplify the target genes and Gapdh.

Tissue sectioning and staining. After incubation, the lenses were immersed in a formalin-glutaraldehyde (FG) solution at $4^{\circ} \mathrm{C}$ for 3 days as described previously ${ }^{56}$. FG solution was exchanged every other day. The lenses were then left in $10 \%$ formalin solution for at least 1 day at room temperature.

Microarray data analysis. All the samples used for microarray were lenses collected at Day 4 . We used a GeneChip Rat Gene 2.0 ST Array (Thermo Fisher Scientific) for microarray analysis. We excluded unnamed genes, and genes with a max signal value below 5 . Each condition's signals were normalized by the galactose-treated sample's signal values. Genes with significant expression changes $(p<0.05)$ in galactose-treated lenses were used for GO term analysis. GO term analysis was performed in the DAVID database (https://david. ncifcrf.gov). Heat mapping was conducted for genes that varied more than twice as much as galactose-treated samples in any one of the respective conditions.

Statistical analysis. Dunnett's t-test was used to determine the difference in averages between Ctrl and experimental groups. $\mathrm{P}<0.05$ was considered statistically significant. The coefficient correlation was calculated by Spearman's method. Statistical analyses were performed using R.

Received: 12 July 2019; Accepted: 6 December 2019;

Published online: 27 December 2019

\section{References}

1. Iwase, A. et al. Prevalence and causes of low vision and blindness in a Japanese adult population: the Tajimi Study. Ophthalmology 113, 1354-1362, https://doi.org/10.1016/j.ophtha.2006.04.022 (2006)

2. Harding, J. J., Egerton, M., van Heyningen, R. \& Harding, R. S. Diabetes, glaucoma, sex, and cataract: analysis of combined data from two case control studies. Br. J. Ophthalmol. 77, 2-6 (1993).

3. Sekimoto, M., Imanaka, Y., Kitano, N., Ishizaki, T. \& Takahashi, O. Why are physicians not persuaded by scientific evidence? A grounded theory interview study. BMC Health Serv. Res. 6, 92, https://doi.org/10.1186/1472-6963-6-92 (2006).

4. Cheng, H. M. \& González, R. G. The effect of high glucose and oxidative stress on lens metabolism, aldose reductase, and senile cataractogenesis. Metabolism 35, 10-14 (1986).

5. Srivastava, S. K. \& Ansari, N. H. Prevention of sugar-induced cataractogenesis in rats by butylated hydroxytoluene. Diabetes 37 , 1505-1508 (1988).

6. Kinoshita, J. H. Mechanisms initiating cataract formation. Proctor Lecture. Invest. Ophthalmol. 13, 713-724 (1974).

7. Li, W. C. et al. Lens epithelial cell apoptosis appears to be a common cellular basis for non-congenital cataract development in humans and animals. J. Cell Biol. 130, 169-181 (1995). 
8. Takamura, Y., Kubo, E., Tsuzuki, S. \& Akagi, Y. Apoptotic cell death in the lens epithelium of rat sugar cataract. Exp. Eye Res. 77, 51-57, https://doi.org/10.1016/s0014-4835(03)00083-6 (2003).

9. Tough, D. F., Tak, P. P., Tarakhovsky, A. \& Prinjha, R. K. Epigenetic drug discovery: breaking through the immune barrier. Nat. Rev. Drug Discov. 15, 835-853, https://doi.org/10.1038/nrd.2016.185 (2016).

10. Suzuki, M. M. \& Bird, A. DNA methylation landscapes: provocative insights from epigenomics. Nat. Rev. Genet. 9, 465-476, https:// doi.org/10.1038/nrg2341 (2008).

11. Li, B. et al. Relationship Between the Altered Expression and Epigenetics of GSTM3 and Age-Related Cataract. Invest Ophthalmol Vis Sci 57, 4721-4732, https://doi.org/10.1167/iovs.16-19242 (2016).

12. Palsamy, P., Ayaki, M., Elanchezhian, R. \& Shinohara, T. Promoter demethylation of Keap1 gene in human diabetic cataractous lenses. Biochem. Biophys. Res. Commun. 423, 542-548, https://doi.org/10.1016/j.bbrc.2012.05.164 (2012).

13. Zhu, X. J. et al. Epigenetic regulation of $\alpha \mathrm{A}$-crystallin in high myopia-induced dark nuclear cataract. PLoS One 8, e81900, https:// doi.org/10.1371/journal.pone.0081900 (2013).

14. Wang, Y., Li, F., Zhang, G. W., Kang, L. H. \& Guan, H. J. Ultraviolet-B induces ERCC6 repression in lens epithelium cells of agerelated nuclear cataract through coordinated DNA hypermethylation and histone deacetylation. Clin. Epigenetics 8, https://doi. org/10.1186/s13148-016-0229-y (2016).

15. Wang, Y. et al. Altered DNA Methylation and Expression Profiles of 8-Oxoguanine DNA Glycosylase 1 in Lens Tissue from Agerelated Cataract Patients. Curr. Eye Res. 40, 815-821, https://doi.org/10.3109/02713683.2014.957778 (2015).

16. Timmermann, S., Lehrmann, H., Polesskaya, A. \& Harel-Bellan, A. Histone acetylation and disease. Cell. Mol. Life Sci. 58, 728-736, https://doi.org/10.1007/pl00000896 (2001).

17. Rong, X. F. et al. Effects of histone acetylation on superoxide dismutase 1 gene expression in the pathogenesis of senile cataract. Sci. Rep. 6, https://doi.org/10.1038/srep34704 (2016).

18. Xie, L., Santhoshkumar, P., Reneker, L. W. \& Sharma, K. K. Histone deacetylase inhibitors trichostatin A and vorinostat inhibit TGFß2-induced lens epithelial-to-mesenchymal cell transition. Invest Ophthalmol Vis Sci 55, 4731-4740, https://doi.org/10.1167/ iovs.14-14109 (2014).

19. Chen, X. et al. The epigenetic modifier trichostatin A, a histone deacetylase inhibitor, suppresses proliferation and epithelialmesenchymal transition of lens epithelial cells. Cell Death Dis. 4, https://doi.org/10.1038/cddis.2013.416 (2013).

20. Dancy, B. M. \& Cole, P. A. Protein Lysine Acetylation by p300/CBP. Chem. Rev. 115, 2419-2452, https://doi.org/10.1021/cr500452k (2015).

21. Simon, R. P., Robaa, D., Alhalabi, Z., Sippl, W. \& Jung, M. KATching-Up on Small Molecule Modulators of Lysine Acetyltransferases. J. Med. Chem. 59, 1249-1270, https://doi.org/10.1021/acs.jmedchem.5b01502 (2016).

22. Ghizzoni, M. et al. 6-alkylsalicylates are selective Tip60 inhibitors and target the acetyl-CoA binding site. Eur. J. Med. Chem. 47, 337-344, https://doi.org/10.1016/j.ejmech.2011.11.001 (2012).

23. Chimenti, F. et al. A Novel Histone Acetyltransferase Inhibitor Modulating Gcn5 Network: Cyclopentylidene- 4-(4 '-chlorophenyl) thiazol-2-yl)hydrazone. J. Med. Chem. 52, 530-536, https://doi.org/10.1021/jm800885d (2009).

24. Choi, K. C. et al. Gallic Acid Suppresses Lipopolysaccharide-Induced Nuclear Factor-kappa B Signaling by Preventing RelA Acetylation in A549 Lung Cancer Cells. Mol. Cancer Res. 7, 2011-2021, https://doi.org/10.1158/1541-7786.mcr-09-0239 (2009).

25. Chekler, E. L. P. et al. Transcriptional Profiling of a Selective CREB Binding Protein Bromodomain Inhibitor Highlights Therapeutic Opportunities. Chem. Biol. 22, 1588-1596, https://doi.org/10.1016/j.chembiol.2015.10.013 (2015).

26. Hao, J. J. et al. Selective Small Molecule Targeting beta-Catenin Function Discovered by In Vivo Chemical Genetic Screen. Cell Rep. 4, 898-904, https://doi.org/10.1016/j.celrep.2013.07.047 (2013).

27. Larrieu, D., Britton, S., Demir, M., Rodriguez, R. \& Jackson, S. P. Chemical Inhibition of NAT10 Corrects Defects of Laminopathic Cells. Science 344, 527-532, https://doi.org/10.1126/science.1252651 (2014).

28. Costi, R. et al. Cinnamoyl compounds as simple molecules that inhibit $\mathrm{p} 300$ histone acetyltransferase. J. Med. Chem. 50, 1973-1977, https://doi.org/10.1021/jm060943s (2007).

29. Mai, A. et al. Epigenetic multiple ligands: Mixed Histone/Protein methyltransferase, acetyltransferase, and class III deacetylase (Sirtuin) inhibitors. J. Med. Chem. 51, 2279-2290, https://doi.org/10.1021/jm701595q (2008).

30. Coffey, K. et al. Characterisation of a Tip60 Specific Inhibitor, NU9056, in Prostate Cancer. PLoS One 7, https://doi.org/10.1371/ journal.pone.0045539 (2012).

31. Sbardella, G. et al. Identification of long chain alkylidenemalonates as novel small molecule modulators of histone acetyltransferases. Biorg. Med. Chem. Lett. 18, 2788-2792, https://doi.org/10.1016/j.bmcl.2008.04.017 (2008).

32. Xie, S. et al. Reactive oxygen species-induced phosphorylation of $\mathrm{p} 53$ on serine 20 is mediated in part by polo-like kinase-3. J. Biol. Chem. 276, 36194-36199, https://doi.org/10.1074/jbc.M104157200 (2001).

33. Xie, S. Q. et al. Plk3 functionally links DNA damage to cell cycle arrest and apoptosis at least in part via the p53 pathway. J. Biol. Chem. 276, 43305-43312, https://doi.org/10.1074/jbc.M106050200 (2001).

34. Whitson, J. A. et al. Transcriptome of the GSH-Depleted Lens Reveals Changes in Detoxification and EMT Signaling Genes, Transport Systems, and Lipid Homeostasis. Invest. Ophthalmol. Vis. Sci. 58, 2666-2684, https://doi.org/10.1167/iovs.16-21398 (2017).

35. Zhou, Y., Bennett, T. M. \& Shiels, A. Lens ER-stress response during cataract development in Mip-mutant mice. Biochim. Biophys. Acta 1862, 1433-1442, https://doi.org/10.1016/j.bbadis.2016.05.003 (2016).

36. Wang, L., Dai, W. \& Lu, L. Stress-induced c-Jun activation mediated by polo-like kinase 3 in corneal epithelial cells. J. Biol. Chem. 282, 32121-32127, https://doi.org/10.1074/jbc.M702791200 (2007).

37. Xie, S. Q. et al. Genotoxic Stress-Induced Activation of Plk3 is Partly Mediated by Chk2. Cell Cycle 1, 424-429, https://doi. org/10.4161/cc.1.6.271 (2002).

38. Kronschläger, M. et al. Caffeine eye drops protect against UV-B cataract. Exp. Eye Res. 113, 26-31, https://doi.org/10.1016/j. exer.2013.04.015 (2013)

39. Varma, S. D., Hegde, K. R. \& Kovtun, S. UV-B-Induced Damage to the Lens In Vitro: Prevention by Caffeine. J. Ocul. Pharmacol. Ther. 24, 439-444, https://doi.org/10.1089/jop.2008.0035 (2008).

40. Wang, L., Dai, W. \& Lu, L. Osmotic Stress-induced Phosphorylation of H2AX by Polo-like Kinase 3 Affects Cell Cycle Progression in Human Corneal Epithelial Cells. J. Biol. Chem. 289, 29827-29835, https://doi.org/10.1074/jbc.M114.597161 (2014).

41. Kador, P. F., Akagi, Y. \& Kinoshita, J. H. The effect of aldose reductase and its inhibition on sugar cataract formation. Metabolism 35, 15-19(1986).

42. Lovicu, F. J. et al. TGFbeta induces morphological and molecular changes similar to human anterior subcapsular cataract. Br. J. Ophthalmol. 86, 220-226 (2002).

43. Kim, Y. H., Kim, Y. S., Roh, G. S., Choi, W. S. \& Cho, G. J. Resveratrol blocks diabetes-induced early vascular lesions and vascular endothelial growth factor induction in mouse retinas. Acta Ophthalmol 90, e31-37, https://doi.org/10.1111/j.1755-3768.2011.02243.x (2012).

44. Vrba, L., Junk, D. J., Novak, P. \& Futscher, B. W. p53 induces distinct epigenetic states at its direct target promoters. BMC Genomics 9, https://doi.org/10.1186/1471-2164-9-486 (2008).

45. Watanabe, M. et al. Regulation of smooth muscle cell differentiation by AT-rich interaction domain transcription factors Mrf2 alpha and Mrf2 beta. Circ. Res. 91, 382-389, https://doi.org/10.1161/01.res.0000033593.05545.7b (2002). 
46. Baba, A. et al. PKA-dependent regulation of the histone lysine demethylase complex PHF2-ARID5B. Nat. Cell Biol. 13, 668-U101, https://doi.org/10.1038/ncb2228 (2011).

47. Whitson, R. H., Tsark, W., Huang, T. H. \& Itakura, K. Neonatal mortality and leanness in mice lacking the ARID transcription factor Mrf-2. Biochem. Biophys. Res. Commun. 312, 997-1004, https://doi.org/10.1016/j.bbrc.2003.11.026 (2003).

48. Bricambert, J. et al. The histone demethylase Phf2 acts as a molecular checkpoint to prevent NAFLD progression during obesity. Nature Communications 9, https://doi.org/10.1038/s41467-018-04361-y (2018).

49. Gearing, D. P. et al. Molecular cloning and expression of cDNA encoding a murine myeloid leukaemia inhibitory factor (LIF). EMBO J. 6, 3995-4002 (1987).

50. Marshall, M. K., Doerrler, W., Feingold, K. R. \& Grunfeld, C. Leukemia Inhibitory Factor Induces Changes in Lipid-Metabolism in Cultured Adipocytes. Endocrinology 135, 141-147, https://doi.org/10.1210/en.135.1.141 (1994).

51. Nonogaki, K. et al. LIF and CNTF, which share the gp130 transduction system, stimulate hepatic lipid metabolism in rats. Am. J. Physiol. Endocrinol. Metabol. 271, E521-E528 (1996).

52. Zhu, H., Cong, J. P. \& Shenk, T. Use of differential display analysis to assess the effect of human cytomegalovirus infection on the accumulation of cellular RNAs: Induction of interferon-responsive RNAs. Proc. Natl. Acad. Sci. USA 94, 13985-13990, https://doi. org/10.1073/pnas.94.25.13985 (1997).

53. Dogan, A. et al. ATR-FTIR spectroscopy reveals genomic loci regulating the tissue response in high fat diet fed BXD recombinant inbred mouse strains. BMC Genomics 14, https://doi.org/10.1186/1471-2164-14-386 (2013).

54. Qi, Z. T. et al. Targeting viperin improves diet-induced glucose intolerance but not adipose tissue inflammation. Oncotarget 8 , 101418-101436, https://doi.org/10.18632/oncotarget.20724 (2017).

55. Sivak, J. G., Yoshimura, M., Weerheim, J. \& Dovrat, A. Effect of hydrogen peroxide, DL-propranolol, and prednisone on bovine lens optical function in culture. Invest. Ophthalmol. Vis. Sci. 31, 954-963 (1990).

56. Sakai, H., Takata, K., Fukuda, M., Fujiwara, T. \& Hirano, H. An improved fixation method for transmission electron microscopy for the histological study of the lens. Okajimas Folia Anat. Jpn. 68, 295-298 (1991).

\section{Acknowledgements}

We thank Dr. Daisuke Kaida for his critical advice. We thank Dr. Kazuhiro Karaya for microarray analysis support. This study was supported by Grant of Life Science Innovation Center (University of Fukui).

\section{Author contributions}

F.K., Y.T., S.M., M. Inami, and M.O. wrote the manuscript. F.K., Y.T., S.M., M. Inatani, and M.O. conceived and designed the study. F.K., K.K., and M. Inami. performed data analysis. All authors read and approved the manuscript.

\section{Competing interests}

The authors declare no competing interests.

\section{Additional information}

Supplementary information is available for this paper at https://doi.org/10.1038/s41598-019-56414-x.

Correspondence and requests for materials should be addressed to M.O.

Reprints and permissions information is available at www.nature.com/reprints.

Publisher's note Springer Nature remains neutral with regard to jurisdictional claims in published maps and institutional affiliations.

Open Access This article is licensed under a Creative Commons Attribution 4.0 International License, which permits use, sharing, adaptation, distribution and reproduction in any medium or format, as long as you give appropriate credit to the original author(s) and the source, provide a link to the Creative Commons license, and indicate if changes were made. The images or other third party material in this article are included in the article's Creative Commons license, unless indicated otherwise in a credit line to the material. If material is not included in the article's Creative Commons license and your intended use is not permitted by statutory regulation or exceeds the permitted use, you will need to obtain permission directly from the copyright holder. To view a copy of this license, visit http://creativecommons.org/licenses/by/4.0/.

(c) The Author(s) 2019 